\title{
Native Defects and their Doping Response in the Lithium Solid Electrolyte $\mathrm{Li}_{7} \mathrm{La}_{3} \mathrm{Zr}_{2} \mathrm{O}_{12}$
}

\author{
Alexander G. Squires, ${ }^{1,2}$ David O. Scanlon, ${ }^{3,4,2,5}$ and Benjamin J. Morgan ${ }^{1,2, *}$ \\ ${ }^{1}$ Department of Chemistry, University of Bath, Bath, BA2 7AX. \\ ${ }^{2}$ The Faraday Institution, Quad One, Harwell Science and Innovation Campus, Didcot, UK. \\ ${ }^{3}$ Department of Chemistry, University College London, 20 Gordon Street, London, WC1H 0AJ. \\ ${ }^{4}$ Diamond Light Source Ltd., Diamond House, Harwell Science and Innovation Campus, \\ Didcot, Oxfordshire OX11 ODE, United Kingdom. \\ ${ }^{5}$ Thomas Young Centre, University College London, Gower Street, London WC1E 6BT, UK
}

(Dated: October 22, 2019)

\begin{abstract}
The Li-stuffed garnets $\mathrm{Li}_{x} M_{2} M_{3}^{\prime} \mathrm{O}_{12}$ are promising Li-ion solid electrolytes with potential use in solid-state batteries. One strategy for optimising ionic conductivities in these materials is to tune lithium stoichiometries through aliovalent doping, which is often assumed to produce proportionate numbers of charge-compensating Li vacancies. The native defect chemistry of the Li-stuffed garnets, and their response to doping, however, are not well understood, and it is unknown to what degree a simple vacancy-compensation model is valid. Here, we report hybrid density-functional-theory calculations of a broad range of native defects in the prototypical Li-garnet $\mathrm{Li}_{7} \mathrm{La}_{3} \mathrm{Zr}_{2} \mathrm{O}_{12}$. We calculate equilibrium defect concentrations as a function of synthesis conditions, and model the response of these defect populations to extrinsic doping. We predict a rich defect chemistry that includes $\mathrm{Li}$ and $\mathrm{O}$ vacancies and interstitials, and significant numbers of cation-antisite defects. Under reducing conditions, $\mathrm{O}$ vacancies act as colour-centres by trapping electrons. We find that supervalent (donor) doping does not produce charge compensating $\mathrm{Li}$ vacancies under all synthesis conditions; under Li-rich / Zr-poor conditions the dominant compensating defects are $\mathrm{Li}_{\mathrm{Zr}}$ antisites, and $\mathrm{Li}$ stoichiometries strongly deviate from those predicted by simple "vacancy compensation" models.
\end{abstract}

\section{INTRODUCTION}

A global transition to low-carbon primary energy sources, such as solar and wind, requires complementary advances in effective secondary-energy-storage technologies. Lithium-ion batteries are a hugely successful energy-storage technology, but the performance of commercial batteries is limited by the use of conventional liquid electrolytes. These contain flammable organic solvents with narrow electrochemical stability windows, which presents a safety risk and precludes the use of energy-dense high-voltage electrodes. ${ }^{1}$ One proposal to address these issues is to replace conventional liquid electrolytes with electrochemically stable, non-flammable ceramics, to allow the development of all-solid-state batteries with higher energy densities and improved safety characteristics. $^{2-7}$ An ideal lithium-ion solid electrolyte should have a wide electrochemical stability window, a negligible electronic conductivity, and a high ionic conductivity. ${ }^{8}$ While a number of families of promising solid lithium-ion conductors are known, ${ }^{2}$ it is generally unknown to what extent these target properties, such as high ionic conductivities, can be achieved by chemical tuning: for example, by doping with chemical substituents or by varying synthesis conditions.

One promising family of solid lithium-ion electrolytes are the lithium-stuffed garnets, $\mathrm{Li}_{x} M_{3} M_{2}^{\prime} \mathrm{O}_{12} \cdot{ }^{3,9}$ These materials have ionic conductivities reportedly as high as $\sim 1 \times 10^{-3} \mathrm{~S} / \mathrm{cm}^{10}$ and wide electrochemical stability windows, ${ }^{11}$ allowing their incorporation in battery systems. ${ }^{12-15}$ By varying the cations $M$ and $M^{\prime}$, many different compositions can be synthesised, with a broad range of ionic conductivities. ${ }^{3}$ Identifying chemical compositions with optimal conductivities, however, remains a challenge, and requires understanding both latticecation substitution and lithium stoichiometry, and how these compositional parameters together affect lithium transport. ${ }^{16-21}$

One strategy for optimising the ionic conductivity of the lithium-conducting garnets is aliovalent doping, which is often assumed to allow control of lithium stoichiometry through the formation of chargecompensating lithium defects. ${ }^{22}$ Aliovalent doping of garnets gained interest following the inadvertent Al-doping of $\mathrm{Li}_{7} \mathrm{La}_{3} \mathrm{Zr}_{2} \mathrm{O}_{12}$ (LLZO), which produced a dramatic increase in room-temperature ionic conductivity by stabilising the highly conducting cubic phase ${ }^{23,24}$ — normally only observed at temperatures above $600 \mathrm{~K}$ for stoichiometric LLZO - with respect to the poorly conducting tetragonal phase. ${ }^{25,26}$ This effect was explained by aluminium substituting for lithium, ${ }^{27}$ causing chargecompensating lithium vacancies to form, with this change in lithium stoichiometry stabilising the cubic phase. ${ }^{19,28}$ Subsequent efforts to optimise the ionic conductivity of doped LLZO have seen a number of supervalent (donor) dopants proposed. ${ }^{29}$ These include other small cations, such as gallium, that directly substitute lithium; ${ }^{30-32}$ larger cations, such as tantalum or niobium, that substitute zirconium or lanthanum on the $M$ or $M^{\prime}$ sites; $^{10}$ and donor anions, such as fluorine, that substitute oxygen. ${ }^{33}$

Donor doping is usually assumed to affect lithium stoichiometry by causing the formation of charge- 
compenstating lithium vacancies, e.g. for a trivalent cation such as $\mathrm{Al}^{3+}$ substituting for monovalent $\mathrm{Li}^{+}$, charge neutrality considerations suggest that ${ }^{31,32}$

$$
\left[\mathrm{Al}_{\mathrm{Li}}^{\bullet \bullet}\right]=2\left[V_{\mathrm{Li}}^{\prime}\right]
$$

(using Kröger-Vink notation, where $A_{B}^{q}$ represents species $A$ at site $B$, with relative charge $q$ ). ${ }^{34}$ Although this direct-charge-compensation model has an attractive conceptual simplicity, it ignores other defect species that may form under specific synthesis conditions or in response to doping. A number of studies have suggested that oxygen vacancies may play a significant role in the defect chemistry of LLZO, ${ }^{18,35-37}$ and Kubicek et al. have confirmed the presence of oxygen vacancies in a range of nominal "LLZO" compositions, using isotope-exchange techniques, with estimated O-vacancy concentrations as high as $2.5 \%{ }^{36}$ Because oxygen vacancies can act as electronic donors, their presence has been proposed to affect lithium stoichiometries through a charge-compensating "Schottky pair" mechanism:

$$
2 \mathrm{Li}_{\mathrm{Li}}^{\times}+\mathrm{O}_{\mathrm{O}}^{\times} \rightleftharpoons 2 V_{\mathrm{Li}}^{\prime}+V_{\mathrm{O}}^{\bullet \bullet} .
$$

This defect equilibrium suggests that synthesis conditions that promote oxygen vacancy formation; e.g. high temperatures or low oxygen-partial-pressures; may produce samples with reduced lithium stoichiometries. Furthermore, the appearance of $V_{\mathrm{Li}}^{\prime}$ in both Eqns. 1 and 2 raises the possibility of coupling between the intrinsic defect chemistry of lithium-stuffed garnets and their response to donor doping. ${ }^{38}$

The example above considers only three defect species, yet illustrates the difficulty in using simple chargecompensation models to understand how defect concentrations are related. In practice, other native defects may also be present in significant numbers, and these should also be included in any thermodynamic model of defect populations. In materials such as LLZO, cationic lattice defects, such as $\mathrm{La}$ and $\mathrm{Zr}$ vacancies, are often assumed to be negligible; ${ }^{37}$ but this is not a priori guaranteed under all synthesis conditions. In addition, simple defect-charge-compensation models usually assume defects exist in only one formal charge state e.g. -1 for $\mathrm{Li}$ vacancies, or +2 for $\mathrm{O}$ vacancies-39 when in reality defects may adopt a range of charge states as a function of Fermi energy. ${ }^{40-43}$ A more complete thermodynamic defect model should therefore consider a broad range of native defects in all accessible charge states. The defect chemistry and doping response are now not described by a single defect-charge-compensation equation, such as Eqns. 1 or 2, but instead by a set of equations that describe the defect populations, to be solved self-consistently under the constraint of thermodynamic equilibrium. ${ }^{40,44-46}$

To better understand the native defect chemistry and doping response of lithium-garnet solid-electrolytes, we have performed a computational study of a broad range of defects in the prototypical system LLZO. We have used hybrid density functional theory (DFT) to calculate formation energies for a range of intrinsic defects, including lithium and oxygen vacancies and interstitials, lanthanum and zirconium vacancies, and cation anti-sites. These defect formation energies are used to construct a self-consistent thermodynamic model of defect concentrations as a function of synthesis conditions. We find a rich defect chemistry that includes not just lithium and oxygen defects, but also significant numbers of cationantisite defects. Oxygen vacancies exhibit $0,+1$, and +2 charge states, and under reducing conditions act as colour-centres by trapping electrons. We have also modelled the response to supervalent (donor) doping, and find that lithium vacancies are not the dominant compensating acceptor defects under all synthesis conditions; under Li-rich / Zr-poor conditions donor doping is primarily compensated by $\mathrm{Li}_{\mathrm{Zr}}$ antisites, and lithium stoichiometries can strongly deviate from the values predicted by the simple "vacancy compensation" model (Eqn. 1).

\section{METHODS}

At thermodynamic equilibrium, the concentration of a defect $X$ in charge-state $q$ is given by

$$
\left[X^{q}\right]=N_{X} g_{X, q} \exp \left(\frac{-\Delta E_{\mathrm{f}}^{X^{q}}}{\mathrm{k} T}\right),
$$

where $N_{X}$ is the density of sites available to form $X$, $g_{X, q}$ is the degeneracy of the defect state (e.g. spin degeneracy), $\mathrm{k}$ is the Boltzmann constant, and $T$ is the temperature. $\Delta E_{\mathrm{f}}^{X^{q}}$ is the free energy of formation of defect $X$ in charge-state $q$. For defects with $q \neq 0$, the formation energy depends on the Fermi energy, i.e. the electron chemical potential. Because the energy to add or remove electrons depends on the defects present, calculating equilibrium defect concentrations consists of finding a self-consistent solution to Eqn. 3 for all defect species, under the constraint of overall charge neutrality. The net charge density at a given Fermi energy, $\rho\left(E_{\mathrm{F}}\right)$, is given by a sum over all charged defects, plus contributions from electrons and holes occupying conduction and valence bands respectively:

$$
\rho\left(E_{\mathrm{F}}\right)=\sum_{X^{q}} q\left[X^{q}\right]+p_{0}-n_{0} .
$$

Electron and hole concentrations ( $n_{0}$ and $p_{0}$ respectively) are given by

$$
\begin{aligned}
& n_{0}=\int_{0}^{\infty} \frac{1}{\mathrm{e}^{\left(E-E_{\mathrm{F}}\right) / \mathrm{k} T}+1} g(E) \mathrm{d} E, \\
& p_{0}=\int_{0}^{\infty} 1-\frac{1}{\mathrm{e}^{\left(E-E_{\mathrm{F}}\right) / \mathrm{k} T}+1} g(E) \mathrm{d} E,
\end{aligned}
$$

where $g(E)$ is the density of states (DOS) for the system under consideration. 
Thermodynamic defect concentrations under extrinsic doping can be calculated by including fixed concentrations of each dopant species and similarly solving to find the Fermi energy that gives overall charge neutrality. Introducing an aliovalent dopant adds, or removes, electrons from the system, depending on the effective relative charge of the resulting defect. For the case of a dopant defect $M$, with relative charge $r$ and fixed concentration $\left[M^{r}\right]$, the net charge density, $\rho$, is given by

$$
\rho\left(E_{\mathrm{F}}, r\left[M^{r}\right]\right)=\sum_{X^{q}} q\left[X^{q}\right]+p_{0}-n_{0}+r\left[M^{r}\right] .
$$

To restore charge neutrality, the native defect concentrations necessarily change from those in the undoped system. This doping response does not depend explicitly on the dopant species and insertion site, but only on the product $r\left[M^{r}\right]$. The response predicted for an effective +2 dopant, e.g. $\mathrm{Al}_{\mathrm{Li}}^{\bullet \bullet}$, is therefore equivalent to that for an effective +1 dopant, e.g. $\mathrm{Ta}_{\mathrm{Zr}}^{\bullet}$ or $\mathrm{F}_{\mathrm{O}}^{\bullet}$, with a concentration scaling of $\times 2$.

This approach assumes that direct defect-dopant interactions are negligible when compared to the response to the Fermi-level shift arising from aliovalent doping. There is some evidence for explicit dopant-defect interactions in LLZO that vary with the identity of the dopant species. Molecular dynamics simulations by Mottet et al. have revealed changes in the average distribution of $\mathrm{Li}$ ions at sites adjacent to $\mathrm{Mo}_{\mathbf{Z r}}$ dopants, ${ }^{21}$ while DFT calculations by Rettenwander et al. have predicted that introducing $\mathrm{Al}^{3+}$ versus $\mathrm{Ga}^{3+}$ at tetrahedral lithium sites increases the site-occupation energy for $\mathrm{Li}$ at the nearest tetrahedral sites by $2 \mathrm{meV}$ and $12 \mathrm{meV}$ respectively. Daza et al. performed simulations of Aland Ga-doped LLZO at low temperature $(233 \mathrm{~K})$ and observed distinct differences in Li distributions for Al- versus Ga-doping, ${ }^{47}$ although this effect decreased at higher temperature $(313 \mathrm{~K})$. While these effects are potentially significant when considering Li diffusion behaviour in doped systems at typical operating temperatures, under high-temperature synthesis conditions we expect direct dopant-defect interactions to only have a small effect on equilibrium defect populations in as-formed garnets.

We have calculated defect formation energies using periodic hybrid density-functional-theory (DFT), within the Zhang and Northrup formalism, ${ }^{40}$ where the formation energy of defect $X$ in charge state $q$ is given by

$$
\begin{aligned}
\Delta E_{\mathrm{f}}^{X^{q}}= & E_{\mathrm{tot}}^{X^{q}}-E_{\mathrm{tot}}^{\mathrm{bulk}}-\sum_{i} n_{i}\left(\mu_{i}+\Delta \mu_{i}\right) \\
& +q\left(E_{\mathrm{F}}+E_{\mathrm{vbm}}+\Delta V_{\mathrm{pot}}\right)+E_{\mathrm{corr}}^{q} .
\end{aligned}
$$

$E_{\text {tot }}^{X^{q}}$ is the total energy of the defective supercell in charge state $q$, and $E_{\text {tot }}^{\text {bul }}$ is the total energy of the host supercell. $\Delta \mu_{i}$ are chemical potentials of neutral atomic species $i$ that are added to $\left(n_{i}>0\right)$ or removed from $\left(n_{i}<0\right)$ the supercell when forming each defect, and $\mu_{i}$ is the elemental reference energy, calculated for each element in its standard state. $E_{\mathrm{F}}$ is the Fermi energy (electron chemical potential) relative to the valence-band maximum (VBM), and accounts for electrons added to $(q<0)$ or removed from $(q>0)$ the supercell. $E_{\mathrm{vbm}}$ is the DFT-calculated energy of the valence-band maximum of the host system. $\Delta V_{\text {pot }}$ is a potential alignment term to account for differences in background electrostatic potentials between the host and defective supercells, which we calculate as a difference in spatially averaged electrostatic potentials. $E_{\text {corr }}^{q}$ is a correction term accounting for the finite size of the supercell, arising from the electrostsatic interaction between defects and their periodic images. For this study, we have used the correction scheme of Lany and Zunger, ${ }^{48}$ adapted for anisotropic systems by Murphy and Hine. ${ }^{49}$

For our defect calculations, we have considered lithium vacancies and interstitials, $V_{\mathrm{Li}}$ and $\mathrm{Li}_{\mathrm{i}}$; oxygen vacancies and interstitials, $V_{\mathrm{O}}$ and $\mathrm{O}_{\mathrm{i}}$; lanthanum and zirconium vacancies, $V_{\mathrm{La}}$ and $V_{\mathrm{Zr}}$; zirconium interstitials. $\mathrm{Zr}_{\mathrm{i}}$; and a range of cation anti-sites: $\mathrm{LaZr}_{\mathrm{Zr}}, \mathrm{Zr}_{\mathrm{Li}}^{\text {oct }}, \mathrm{Zr}_{\mathrm{Li}}^{\text {tet }}, \mathrm{Zr}_{\mathrm{La}}, \mathrm{Li}_{\mathrm{La}}$, $\mathrm{La}_{\mathrm{Li}}^{\text {oct }}$ and $\mathrm{Li}_{\mathrm{Zr}}$. A superscript oct or tet denotes a defect located at an octahedral or tetrahedral Li site, respectively. Preliminary calculations using the PBEsol functional ${ }^{50}$ gave a difference in formation energy for $V_{\mathrm{Li}}^{\text {oct }}$ and $V_{\mathrm{Li}}^{\text {tet }}$ of $<0.05 \mathrm{eV}$, and we therefore considered these $\mathrm{Li}$ vacancies to be energetically equivalent for our subsequent HSE06 calculations. Structural relaxations for all defects were calculated with cell parameters fixed to the optimised values for stoichiometric LLZO.

Defect formation energies depend on the chemical potentials of the atomic species added to or removed from the system when forming the defect (Eqn. 8). To restrict the available chemical potential space to values that are, in principle, experimentally accessible, we consider only chemical potentials for which LLZO is thermodynamically stable with respect to competing phases. We have considered the set of competing phases identified by Canepa et al..$^{51}$ (see the SI, section S2), and have calculated the thermodynamic stability regime using the code CPLAP. ${ }^{52}$

Density functional theory (DFT) calculations were performed using the plane-wave code VASP, ${ }^{53-56}$ with valence electrons described by a plane-wave basis set with a cutoff of $520 \mathrm{eV}$. Interactions between core and valence electrons were described using the projector-augmented wave (PAW) method, ${ }^{57}$ with cores of $[\mathrm{H}]$ for $\mathrm{Li}$, [Xe] for $\mathrm{La},[\mathrm{Kr}]$ for $\mathrm{Zr}$ and $[\mathrm{He}]$ for O. Optimised lattice parameters for LLZO, competing phases, and elemental references were obtained by performing a series of constant-volume geometry optimisation calculations, and fitting the resulting energy-volume data to the Murnaghan equation of state. ${ }^{58}$

All calculations used the screened hybrid functional HSE06. ${ }^{59}$ HSE06 gives optimised lattice parameters for tetragonal LLZO $\left(a, b=13.00 \AA, c=12.50 \AA, \alpha=90^{\circ}\right)$ that are in good agreement with experimental values $\left(a, b=13.13 \AA, c=12.66 \AA, \alpha=90^{\circ}\right) .{ }^{60,61}$ The HSE06 calculated band gap of $5.9 \mathrm{eV}$ is also in good agreement with experimental values of $\sim 6.0 \mathrm{eV} .{ }^{11}$ HSE06 has also 
been shown to give accurate descriptions of defects in a range of oxides, ${ }^{62-65}$ in contrast to standard GGA functionals, which often give qualitatively incorrect descriptions of defect states in wide-gap oxides ${ }^{66-68}$ (the specific example of the $V_{\mathrm{Li}}^{\times}$defect in LLZO is discussed in more detail in section $\mathrm{S} 1$ of the $\mathrm{SI}$ ).

All calculations of LLZO were performed on the 96 atom primitive cell of the low-temperature tetragonal phase. Although donor-doped LLZO typically adopts a cubic lattice geometry, the tetragonal $\rightarrow$ cubic transition is associated with onset of lithium disorder, ${ }^{28}$ making defect formation energies poorly defined within the formalism of Eqn. $8{ }^{69} k$-point sampling was selected to ensure energies converged to $<1 \mathrm{meV} /$ atom: all LLZO calculations used a $2 \times 2 \times 2$ Monkhorst-Pack $k$-point mesh. $k$-point sampling for competing phases and elemental reference calculations is described in the supporting dataset. ${ }^{70}$

A dataset containing inputs and outputs for all DFT calculations supporting this study is available under the CC-BY-4.0 licence from the University of Bath Research Data Archive. ${ }^{70}$ Python codes for calculating defect formation energies and defect concentrations as functions of elemental chemical potentials, and Jupyter notebooks used to generate Figs. 1, 2, 4, 5, and 6 are available under the MIT licence. ${ }^{71}$ Our analysis codes use the MATPLOTLIB, ${ }^{72}$ NUMPy, ${ }^{73}$ PANDAs, ${ }^{74}$ PyMATGEn, ${ }^{75}$ SCIPY, ${ }^{76}$ TQDM, ${ }^{77}$ and VASPPY ${ }^{78}$ Python packages, and SC-FERMi and Frozen SC-FERMI ${ }^{45,46}$ for calculating self-consistent defect concentrations.

\section{RESULTS}

\section{A. Intrinsic Defect Chemistry}

The predicted region of thermodynamic stability of LLZO spans a range of values that can be broadly characterised along an oxygen-rich/metal-poor to oxygenpoor/metal-rich axis (full details are given in section S2 of the SI). Fig. 1 shows calculated defect concentrations at three illustrative sets of chemical potentials, spanning from oxygen-rich/metal-poor to oxygen-poor/metal-rich conditions. In each case, we also show a defect transitionlevel diagram (bottom panels), which shows defect formation energies as a function of Fermi energy, and the self-consistent Fermi energy, calculated at $1500 \mathrm{~K}$.

At each set of chemical potential conditions we predict high concentrations of a broad range of defects, including lithium vacancies and interstitials, oxygen vacancies and interstitials, and a range of cation-antisites. Under O-rich conditions the defects with the highest concentrations are $V_{\mathrm{Li}}, \mathrm{Li}_{\mathrm{La}}, \mathrm{LaZr}, \mathrm{Li}_{\mathrm{Zr}}$, and $\mathrm{Zr}_{\mathrm{Li}}$. Intermediate chemical potentials give similar defect concentrations, with $\mathrm{Li}$ vacancies and metal anti-sites dominating. Under O-poor/metal-rich conditions the concentration of $V_{\mathrm{O}}$ increases, becoming the dominant defect species. Li vacancy and metal anti-site concentrations, however, remain high.
The analysis above considers a two-dimensional projection of the four-dimensional $\{\mathrm{O}, \mathrm{Li}, \mathrm{La}, \mathrm{Zr}\}$ chemical potential space. At a fixed value of $\Delta \mu_{\mathrm{O}}$, varying the metal chemical potentials can cause a change in the dominant cation defect. For example (SI section 3), moving from Li-rich/Zr-poor to La-poor/Zr-rich conditions at constant $\Delta \mu_{\mathrm{O}}$ causes the dominant antisite defects to change from $\mathrm{Li}_{\mathrm{Zr}}$ and $\mathrm{Li}_{\mathrm{La}}$ to $\mathrm{Zr}_{\mathrm{Li}}$ and $\mathrm{Zr}_{\mathrm{La}}$.

\section{B. Variation in Lithium Stoichiometry}

Tuning the lithium stoichiometry in lithium-stuffed garnets is a popular synthesis strategy to improve their ionic conductivities. ${ }^{10,18,32,79}$ A key question concerning the defect chemistry of these materials, therefore, is to what extent might their lithium stoichiometries differ from formal values as a function of synthesis conditions, due to native defect formation. ${ }^{18}$ As described above, the range of chemical potentials under which LLZO is predicted to be thermodynamically stable can be characterised as lying between oxygen-rich/metal-poor and oxygen-poor/metal-rich limits. Of the component elements, the chemical potential of oxygen is perhaps most sensitive to synthesis conditions, and most easily controlled by varying temperature or oxygen partialpressure during synthesis. Because oxygen vacancies act as donors, it has been suggested that increasing the concentration of $\mathrm{O}$ vacancies could cause a corresponding increase in acceptor defects, e.g. $V_{\mathrm{Li}}$, potentially reducing lithium stoichiometries below their nominal values ${ }^{18,36}$ (e.g. Eqn. 2).

Previous quantitative analyses of the relationship between oxygen vacancies and lithium vacancies have used simple defect models that include only oxygen and lithium defect species ${ }^{18,36,37}$ or only fully charged and neutral defects. ${ }^{39}$ In the previous section, however, we have shown that considering a broad range of defects in LLZO, in all charge states, reveals a more complex defect chemistry (Fig. 1). In particular, cation antisites - which include both donor and acceptor speciesare formed in high concentrations under all thermodynamically accessible synthesis conditions. The presence of these additional defects means the relationship between oxygen and lithium stoichiometries is likely to be more complex than is predicted by simple chargecompensation schemes.

To illustrate the effect of including a "full" set of defects and charge states in our thermodynamic model, we have calculated the lithium vacancy concentration as a function of oxygen chemical potential using two models. The first is a "lithium-oxygen" model, where we include only oxygen vacancies, lithium vacancies, and lithium interstitials. The second is an "all-defects" model that includes all the defects discussed in the previous section. For each model, we have performed a series of self-consistent defect calculations along a line in chemical potential space from O-rich to O-poor conditions (full 

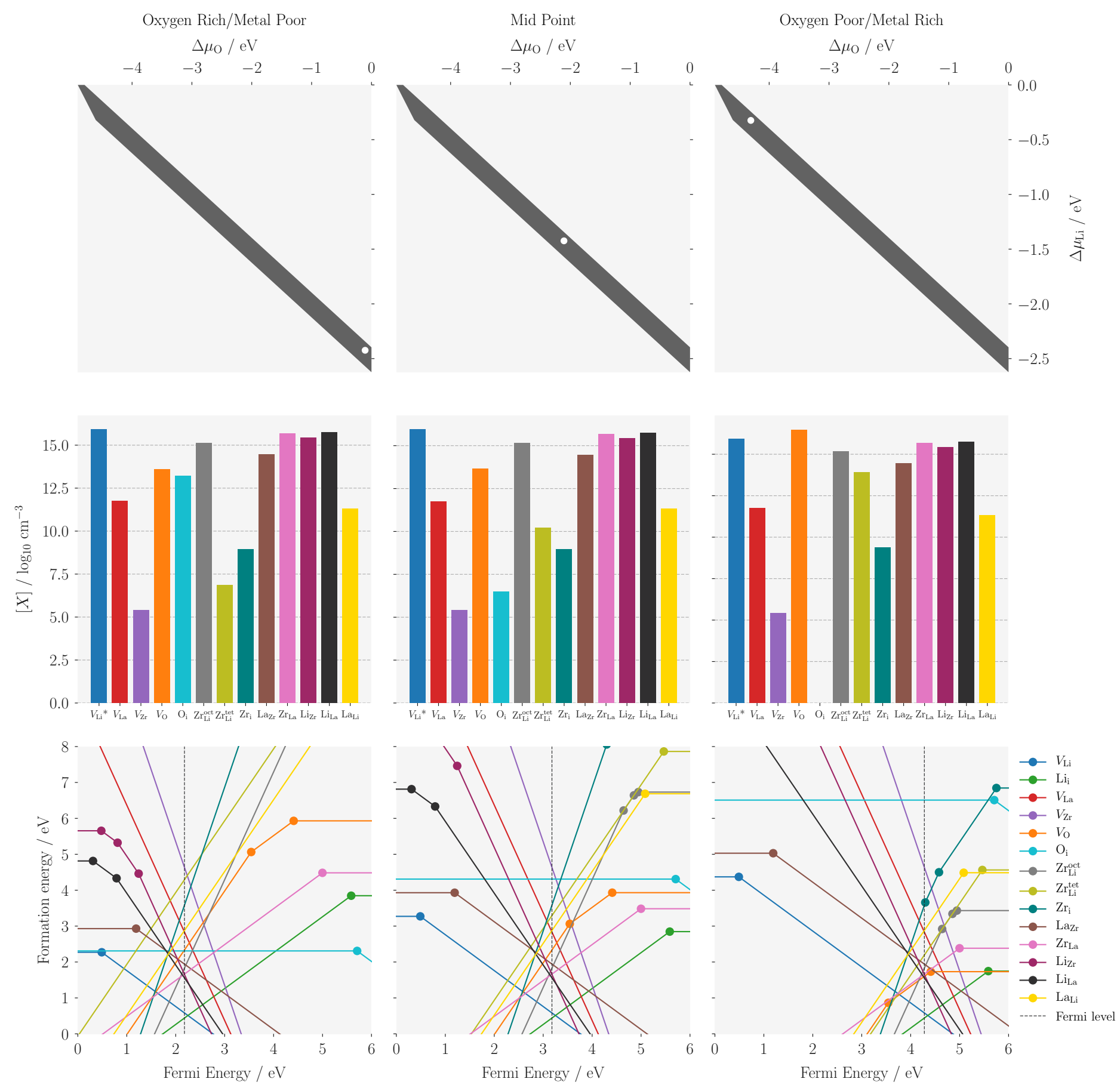

FIG. 1. Equilibrium defect concentrations (middle panels) and defect formation-energies as a function of Fermi energy (bottom panels) at three sets of elemental chemical potentials within the LLZO thermodynamic stability region (top panel). Defect concentrations are calculated at $T=1500 \mathrm{~K}$. In each case, the bottom panel also shows the corresponding self-consistent Fermi energy (vertical dotted line). $\left[V_{\mathrm{Li}}^{*}\right]$ denotes the net lithium vacancy concentration, i.e. $\left[V_{\mathrm{Li}}^{*}\right]=\left[V_{\mathrm{Li}}\right]-\left[\mathrm{Li}_{\mathrm{i}}\right]$. Source: The data and code used to generate this figure, and the figure file, are available under the MIT license as part of Ref. 71.

details are provided in the SI, section S2). The predicted oxygen vacancy and lithium vacancy concentrations in each case are shown in Fig. 2.

The simpler lithium-oxygen model predicts two regimes: at high $\Delta \mu_{\mathrm{O}}$, the concentrations of lithium va- cancies and of oxygen vacancies are both approximately independent of $\Delta \mu_{\mathrm{O}}$, and the ratio of $\left[V_{\mathrm{Li}}\right]:\left[V_{\mathrm{O}}\right]$ is $\sim 2: 1$, as expected from simple charge neutrality arguments (Eqn. 2). The observation that the vacancy concentrations in this regime are independent of $\Delta \mu_{\mathrm{O}}$ can be un- 
derstood by considering the corresponding changes in the defect transition level diagrams (e.g. Figs. 1a \& 1b). Being constrained by the region of thermodynamic stability for LLZO means a decrease in $\Delta \mu_{\mathrm{O}}$ is coupled to an increase in $\Delta \mu_{\mathrm{Li}}$. As $\Delta \mu_{\mathrm{O}}$ decreases, the Fermi energy increases (pinned by the $V_{\mathrm{Li}} / \mathrm{Li}_{\mathrm{i}}$ crossing point). The formation energies of $V_{\mathrm{Li}}, \mathrm{Li}_{\mathrm{i}}$, and $V_{\mathrm{O}}$ (the latter in the +2 charge state) remain unchanged from their values at high $\Delta \mu_{\mathrm{O}}$, and these defect concentrations are therefore also unchanged. At even lower values of $\Delta \mu_{\mathrm{O}}$, a second regime is predicted, in which decreasing $\Delta \mu_{\mathrm{O}}$ causes both $V_{\mathrm{Li}}$ and $V_{\mathrm{O}}$ concentrations to increase. This change in behaviour occurs when the Fermi energy becomes sufficiently high that the lowest energy $V_{O}$ charge state changes from +2 to +1 . Further decreases in $\Delta \mu_{\mathrm{O}}$ are no longer fully compenstated by increases in the Fermi energy, and the $V_{\mathrm{O}}$ energy starts to decrease. Because the dominant $V_{\mathrm{O}}$ charge state in this regime is +1 the $\left[V_{\mathrm{Li}}\right]:\left[V_{\mathrm{O}}\right]$ ratio decreases towards $1: 1$.

The "full" model has a similar overall shape, showing two general regimes of behaviour. The $\Delta \mu_{\mathrm{O}}$-independent regime is broader, however, than for the lithium-oxygen model, and lithium vacancy concentrations are higher, i.e. somewhat decoupled from the $V_{\mathrm{O}}$ concentrations. This is because the model now includes additional donor defects, such as $\mathrm{Zr}_{\mathrm{Li}}^{\cdots}$ and $\mathrm{Zr}_{\mathrm{La}}^{\bullet}$, with low formation energies. The transition to the $\Delta \mu_{\mathrm{O}}$-dependent regime occurs at a lower value of $\Delta \mu_{\mathrm{O}}$ than in the lithium-oxygen model, and oxygen and lithium vacancy concentration are less tightly coupled. This, again, is due to the presence of other defects, particularity cation antisite defects, which buffer the response of system to changing $\Delta \mu_{\mathrm{O}}$. Strong coupling between $V_{\mathrm{O}}$ and $V_{\mathrm{Li}}$ concentrations now happens only when $\Delta \mu_{\mathrm{O}}$ is sufficiently low that $V_{\mathrm{O}}$ is the lowest-energy donor defect.

By performing this analysis across the full fourdimensional thermodynamic stability region of LLZO, we can calculate the full range of variation in lithium stoichiometry under all thermodynamically accessible synthesis conditions. ${ }^{71}$ Although the concentrations of individual defects vary with changing thermodynamic conditions, the net variation in lithium stoichiometry is small. We predict the minimum and maximum lithium stoichiometries of "LLZO" under thermodynamic equilibrium to be $x_{\mathrm{Li}}=6.9975$ and $x_{\mathrm{Li}}=7.00125$.

Lithium stoichiometry is often assumed to directly describe the number of mobile charge-carrying $\mathrm{Li}^{+}$-ions. A simple sum over all lithium-defects does not, however, account for possible differences in mobility for lithium present as different defect species. For example, under Lirich/Zr-poor conditions we predict high concentrations of $\mathrm{Li}_{\mathrm{Zr}}$ antisites. The binding energy of lithium at a zirconium site can be estimated from the "Frenkel-pair" formation energy for $\mathrm{Li}_{\mathrm{Zr}} \rightarrow \mathrm{Li}_{\mathrm{i}}+V_{\mathrm{Zr}}$, which we calculate as $\Delta E=+3.42 \mathrm{eV}$. For comparison the Frenkel-pair formation energy for $\mathrm{Li}_{\mathrm{Li}} \rightarrow \mathrm{Li}_{\mathrm{i}}+V_{\mathrm{Li}}$ is $\Delta E=+1.02 \mathrm{eV}$. The much larger energy cost to remove $\mathrm{Li}$ from a $\mathrm{Zr}$ site, than from a Li site, suggests that lithium present as $\mathrm{Li}_{\mathrm{Zr}}$ (and

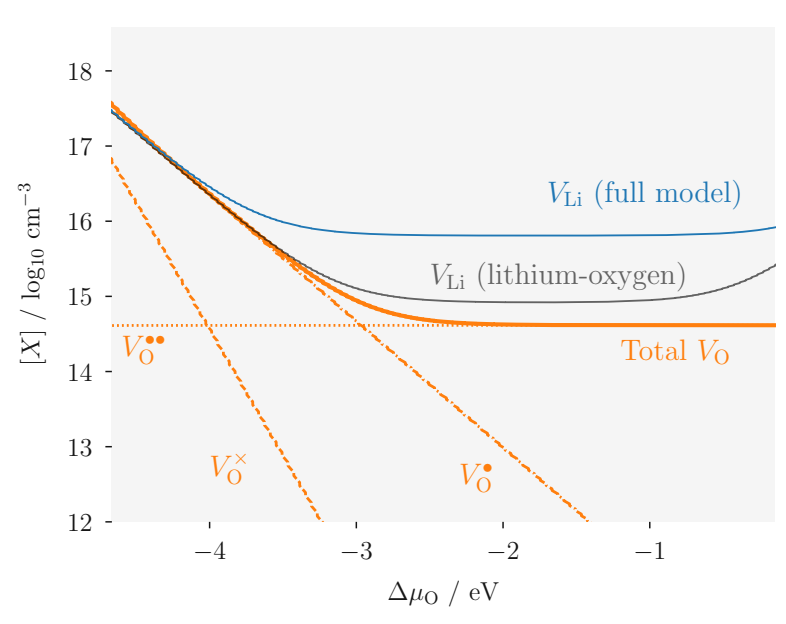

FIG. 2. Predicted $V_{\mathrm{Li}}$ and $V_{\mathrm{O}}$ concentrations as a function of $\Delta \mu_{\mathrm{O}}$, from two comparative models: "lithium-oxygen" model, which only considers $V_{\mathrm{O}}, V_{\mathrm{Li}}$, and $\mathrm{Li}_{\mathrm{i}}$ (all charge states) and "full" model including all defects considered in this paper. Source: The data and code used to generate this figure, and the figure file, are available under the MIT license as part of Ref. 71.

analogously $\mathrm{Li}_{\mathrm{La}}$ ) is strongly bound to the lattice-cation site, and is therefore not available as a mobile chargecarrier. This effect is not, however, expected to significantly affect lithium-ion conductivities, as within the regime of thermodynamic stability the maximum number of $\mathrm{Li}$ ions trapped as $\mathrm{Li}_{\mathrm{Zr}}$ antisites remains a small proportion of the total $\mathrm{Li}(\leq 0.002 \%)$.

A second factor that might effect lithium-ion mobilities is possible clustering of $V_{\mathrm{Li}}^{\prime}$ and $\mathrm{Li}_{\mathrm{i}}^{\bullet}$ to form associated defect pairs. ${ }^{38,39}$ Charged lithium vacancies and interstitials are expected to exhibit a mutual coulombic attraction, giving a favourable negative defect association energy. Formation of a defect complex, however, decreases the number of independent defects in a system, and therefore decreases configurational entropy. Whether an equilibrium population of defects consists predominantly of bound complexes or of independent defects depends on the balance of these energetic and entropic contributions. These thermodynamic considerations give the approximate condition that for defect pairs to be predominantly associated at equilibrium, the defect-pair binding energy, $E_{\mathrm{b}}$, defined as the energy to separate a "bound" defect pair, should be greater than the formation energy, $E_{\mathrm{f}}^{X}$ of the individual defect species. ${ }^{80}$ If the inverse is true, and $E_{\mathrm{f}}^{X}>E_{\mathrm{b}}$, these defects will be predominantly dissociated at equilibrium. To assess this behaviour in LLZO, we have calculated formation energies for $V_{\mathrm{Li}}-\mathrm{Li}_{\mathrm{i}}$ defect pairs separated by $4.16 \AA$ and by $6.13 \AA$. $4.16 \AA$ corresponds to the shortest $V_{\mathrm{Li}}-\mathrm{Li}_{\mathrm{i}}$ distance at which these defects do not simply recombine during geometry optimisation, while $6.13 \AA$ is the largest possible separation in the 96 atom primitive cell. ${ }^{81}$ These calculations give a defect-pair association energy of $0.09 \mathrm{eV}$. Comparing the 
energy of the "associated" defect pair directly with the separate $V_{\mathrm{Li}}^{\prime}$ and $\mathrm{Li}_{\mathrm{i}}^{\bullet}$ formation energies gives a defectpair association energy of $0.19 \mathrm{eV} .{ }^{82}$ Both calculated association energies are much smaller than the $V_{\mathrm{Li}}^{\prime}$ and $\mathrm{Li}_{\mathrm{i}}^{\bullet}$ equilibrium formation energies of $\sim 0.51 \mathrm{eV}$, suggesting that $V_{\mathrm{Li}}$ and $\mathrm{Li}_{\mathrm{i}}$ defects adopt entropy-dominated disordered configurations.

\section{C. $V_{\mathrm{O}}$ Charge States and Oxygen Diffusion}

Although the oxygen-vacancy concentration is not predicted to directly affect the lithium-vacancy concentration, except under extreme O-poor conditions, we do predict a wide range of equilibrium concentrations for oxygen vacancies, varying from a slightly oxygen-rich material $\left(\left[\mathrm{O}_{\mathrm{i}}\right]=2.56 \times 10^{13} / \mathrm{cm}^{3}\right)$ to an oxygen-poor material $\left(\left[V_{\mathrm{O}}\right]=6.53 \times 10^{17} / \mathrm{cm}^{3}\right.$ across the LLZO thermodynamic stability region. $\mathrm{O}$ vacancies have previously been suggested to affect the electronic, optical, and ionconduction properties of lithium-garnets. ${ }^{35,36}$ In this section, we first examine the electronic properties of oxygen vacancies, and then consider their capacity to diffuse through the garnet lattice, thereby potentially contributing to net ionic conductivities.

The simple "Schottky-pair" charge-compenstation model of oxygen vacancy formation (Eqn. 2) considers oxygen vacancies to have a formal charge of +2 . Our calculations predict that at low $\Delta \mu_{\mathrm{O}}$ the favoured oxygenvacancy charge state is +1 , or even 0 ; oxygen vacancy formation leaves behind one, or two, electrons to be accommodated within the host lattice. In oxides containing reducible cations, such as $\mathrm{TiO}_{2}$, excess electrons from donor defects, such as $V_{\mathrm{O}}$, are typically accommodated by reducing these cations (e.g. for $\mathrm{TiO}_{2}$ this formally corresponds to reduction from $\mathrm{Ti}^{4+}$ to $\mathrm{Ti}^{3+66,83}$ ). LLZO does not contain any cations normally considered to have alternate accessible oxidation states, which would normally be associted with undesirable electronic conduction. We find that excess electrons from $V_{\mathrm{O}}$ formation can be trapped at the vacant oxygen site (Fig. 3) to form occupied defect states lying deep in the band gap (Fig. 4), i.e. oxygen vacancies act as F-centers under reducing, or n-type, conditions. This behaviour is consistent with the experimental observations of Wolfenstein et al. who observed colouration of LLZO samples heated in (highly reducing) molten $\mathrm{Li}$, associated with the appearance of a characteristic EPR signal proposed to correspond to unpaired electrons trapped at color centres. ${ }^{35}$

Kubicek et al. have proposed that diffusion of oxygen vacancies in LLZO may contribute to net ionic conductivities. ${ }^{36}$ To evaluate the capacity for diffusion by $V_{\mathrm{O}}$, we have performed climbing-image nudged-elasticband (cNEB) calculations ${ }^{84}$ on the six symmetry inequivalent $V_{\mathrm{O}}$ diffusion pathways between nearest-neighbour O-site pairs (full details are in the SI, section S4). We find the lowest diffusion barrier is $0.73 \mathrm{eV}$, which is similar to the barriers in high-temperature oxide-ion conductors. ${ }^{85,86}$ This suggests oxygen conduction does not make a significant contribution to net ionic conductivities under typical battery operating conditions, in agreement with the experimental analysis of Kubicek et $a l .{ }^{36}$ Oxygen vacancies may, however, diffuse through the host structure during high-temperature sintering. a) $V_{\mathrm{O}}^{\bullet}$

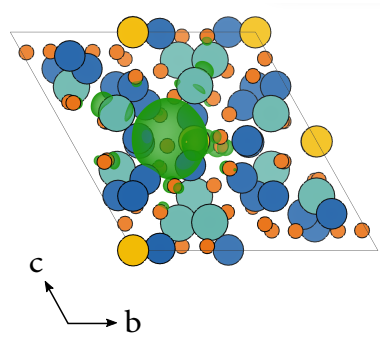

b) $V_{\mathrm{O}}^{\times}$

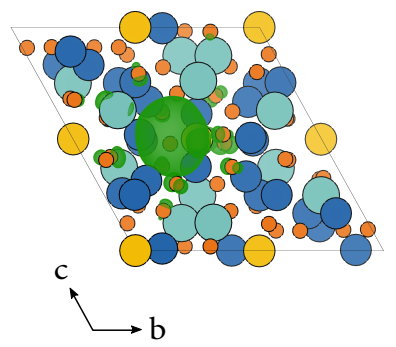

FIG. 3. Partial charge density associated with the F-centre defect states for (a) $V_{\mathrm{O}}^{\bullet}$ and (b) $V_{\mathrm{O}}^{\times}$(B). Oxygen ions are shown in orange, lithium ions in blue, zirconium in yellow, and lanthanum in light blue. The partial charge density is shown in green. Source: The geometry and charge density data used to generate this figure are available under the CCBY-4.0 licence as part of Ref. 70 .

\section{Response to Supervalent Doping}

Having evaluated the native defect chemistry of LLZO as a function of synthesis conditions, we now consider the response to extrinsic doping by supervalent species. The doping-response can be calculated within the selfconsistent defect formalism by calculating the equilibrium concentrations of all native defects, in the presence of a fixed concentration of the relevant extrinsic dopant. ${ }^{46}$ The calculated response to supervalent doping is independent of the choice of dopant species and insertion site, within a concentration scaling factor, as discussed in the Methods section above. Here, we scale the predicted doping response using a generic " $2+$ " dopant, such as a trivalent cation at a lithium site, $M_{\mathrm{Li}}^{\bullet \bullet}$. Within a simple charge compensation model (Eqn. 1) each dopant is expected to produce two charge-compensating lithium vacancies. Fig. 5(a) shows the calculated excess lithium vacancy concentration, relative to the corresponding undoped system, under two sets of chemical potentials. In the first example $\left(\Delta \mu_{\mathrm{Li}}=-1.8 \mathrm{eV}, \Delta \mu_{\mathrm{Zr}}=-6.2 \mathrm{eV}, \Delta \mu_{\mathrm{La}}=\right.$ $\left.-5.2 \mathrm{eV}, \Delta \mu_{\mathrm{O}}=-1.6 \mathrm{eV}\right)$. the doping response exactly follows the prediction of the simple charge-compensation model. Additional lithium vacancies are introduced in a 2:1 ratio to the number of $M_{\mathrm{Li}}$ dopants. In the second example, however, $\left(\Delta \mu_{\mathrm{Li}}=-2.1 \mathrm{eV}, \Delta \mu_{\mathrm{Zr}}=-8.4 \mathrm{eV}\right.$, $\left.\Delta \mu_{\mathrm{La}}=-6.5 \mathrm{eV}, \Delta \mu_{\mathrm{O}}=-0.7 \mathrm{eV}\right)$, the number of excess lithium-vacancies is significantly lower than expected, with a negative deviation from the previous $2: 1$ ratio that becomes larger with dopant concentration. 


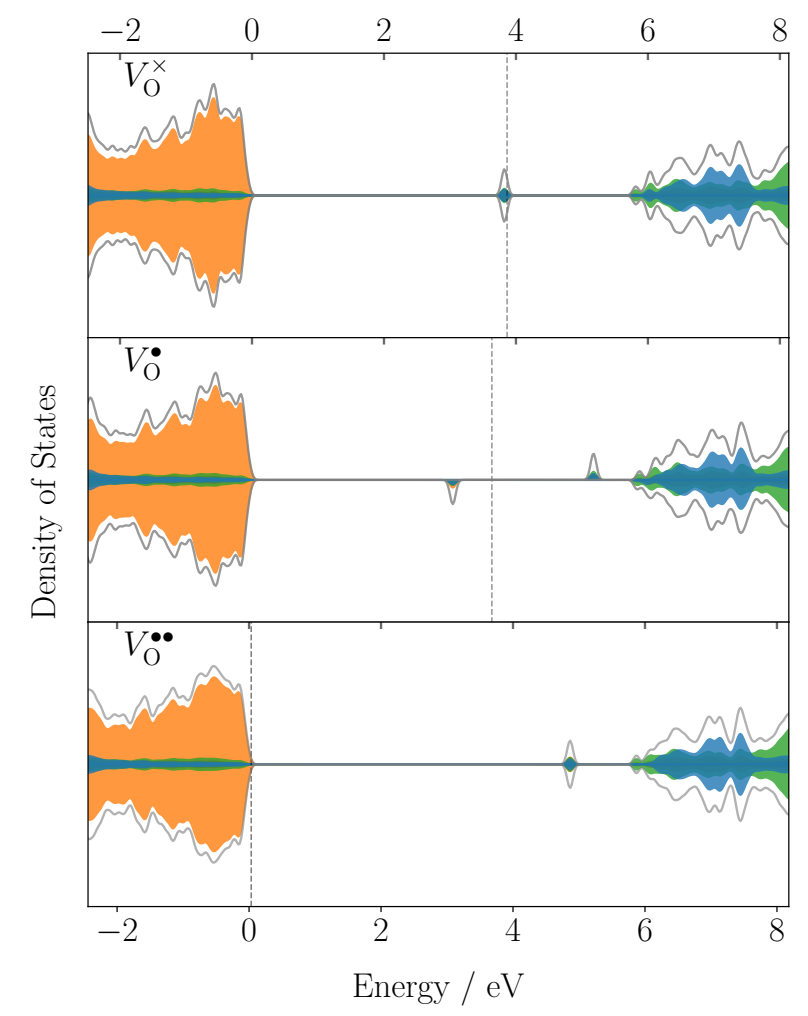

Total DOS
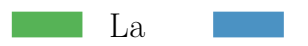

FIG. 4. Projected density of states for oxygen vacancies in $0,+1$, and +2 charge states. The valence-band maximum is aligned to $0 \mathrm{eV}$. The grey line indicates the position of the Fermi level. Source: The data and code used to generate this figure, and the figure file, are available under the MIT licence as part of Ref. 71 .

These contrasting behaviours can be understood by examining the transition level diagrams in each case, and considering the effects of changing the Fermi level when introducing dopants. Figs. $5 \mathrm{~b}$ and $5 \mathrm{c}$, respectively, show the two transition level diagrams for each set of chemical potentials. For clarity we only show the relevant lowest formation-energy defects: $V_{\mathrm{Li}}, \mathrm{Li}_{\mathrm{i}}$, and $\mathrm{Li}_{\mathrm{Zr}}$. These two figures also show the self-consistent Fermi level calculated for the undoped system $\left(\left[M_{\mathrm{Li}}\right]=0\right)$ and for $\left[M_{\mathrm{Li}}\right]=0.15$ formula unit. In both cases, when undoped, the Fermi energy is pinned slightly below the $V_{\mathrm{Li}}$ $/ \mathrm{Li}_{\mathrm{i}}$ crossing point. Adding donor dopants to the system increases the Fermi energy. In the absence of low-energy competing defects (Fig. 5b) this decreases the $V_{\mathrm{Li}}$ energy, and increases the $\mathrm{Li}_{\mathrm{i}}$ energy, resulting in an increased concentration of lithium vacancies. In the second case, the $\Delta \mu_{\mathrm{Li}}$ chemical potential is relatively high compared to $\Delta \mu_{\mathrm{Zr}}$, and increasing the Fermi level through doping causes the $\mathrm{Li}_{\mathrm{Zr}}^{\prime \prime \prime}$ formation energy to fall below that of $V_{\mathrm{Li}}^{\prime}$. For sufficiently high dopant concentrations, therefore, the Fermi energy is shifted high enough that $\mathrm{Li}_{\mathrm{Zr}}$ becomes the dominant acceptor defect. Further donor

doping will now increase the amount of $\mathrm{Li}$ in the system, as the concentration of $\mathrm{Li}_{\mathrm{Zr}}$ increases more rapidly than that of $V_{\mathrm{Li}}$. The significance of the relative values $\Delta \mu_{\mathrm{Li}}$ and $\Delta \mu_{\mathrm{Zr}}$ can be seen in Fig. 6 , which shows the deviation from simple charge-compensating behaviour by $V_{\mathrm{Li}}$ as a function of $\Delta \mu_{\mathrm{Li}}$ and $\Delta \mu_{\mathrm{Zr}}$, calculated for a grid of points across the full region of thermodynamic stability for LLZO. The degree to which lithium vacancy formation is suppressed is broadly described by $\Delta \mu_{\mathrm{Zr}}-4 \Delta \mu_{\mathrm{Li}}$, where the factor of 4 accounts for the difference in charge states between the $\mathrm{Li}_{\mathrm{Zr}}^{\prime \prime \prime}$ and $V_{\mathrm{Li}}^{\prime}$ defects (a derivation of this approximate relation is given in the SI, section $\mathrm{S} 6$ ). The low formation energy of the Lizr defect can be partly ascribed to the similar ionic radii of six-coordinate $\mathrm{Li}^{+}$ $(0.76 \AA)$ and six-coordinate $\mathrm{Zr}^{4+}(0.72 \AA) .{ }^{87}$

\section{SUMMARY AND DISCUSSION}

One strategy for optimising the ionic conductivities of lithium-ion solid electrolytes is to tune the concentration of mobile lithium ions through supervalent doping. ${ }^{10,88-91}$ For quantitative control of lithium stoichiometry to be possible, it is necessary to understand the native defect chemistry of the target material, and how this varies with synthesis conditions, as well as the defectresponse to extrinsic doping. In this study, we have considered these questions for the prototypical lithiumstuffed garnet $\mathrm{Li}_{7} \mathrm{La}_{3} \mathrm{Zr}_{2} \mathrm{O}_{12}$ (LLZO), by performing hybrid density-functional theory calculations on a broad range of defects, and calculating self-consistent defect concentrations as a function of synthesis conditions (component chemical potentials) and dopant concentrations.

For the native defects, we find a rich family of defect species, including lithium and oxygen vacancies and interstitials, which have been discussed previously, ${ }^{36-39}$ and cation anti-sites; such as $\mathrm{Li}_{\mathrm{La}}, \mathrm{La}_{\mathrm{Zr}}, \mathrm{Li}_{\mathrm{Zr}}$, and $\mathrm{Zr}_{\mathrm{Li}}$; which are often neglected when considering the defect chemistry of lithium-garnets. Under all conditions except extremely O-poor (reducing) conditions, cation anti-site defects are the highest concentration defect species after $V_{\mathrm{Li}}$. The existence of multiple native donor and acceptor defect species means the net lithium stoichiometry is somewhat insensitive to synthesis conditions. We predict that in undoped LLZO under equilibrium conditions the lithium stoichiometry deviates from its nominal value of $x_{\mathrm{Li}}=7$ by only $+0.00125 /-0.0025$. Not all this lithium is expected to equally contribute to lithium conduction, however. Under Li-rich conditions we predict high concentrations of $\mathrm{Li}_{\mathrm{Zr}}$ and $\mathrm{Li}_{\mathrm{La}}$ antisites, which are expected to contain strongly bound, immobile, lithium.

Under strongly reducing conditions, $\mathrm{O}$ vacancies are stable in +1 or neutral (0) charge states, and act as colour-centres by trapping electrons. We predict the lowest barrier for oxygen vacancy diffusion is $0.73 \mathrm{eV}$, which suggests that vacancy-mediated oxygen conduction is not significant at typical battery-operating temperatures, in agreement with previous experimental analysis; ${ }^{36}$ al- 

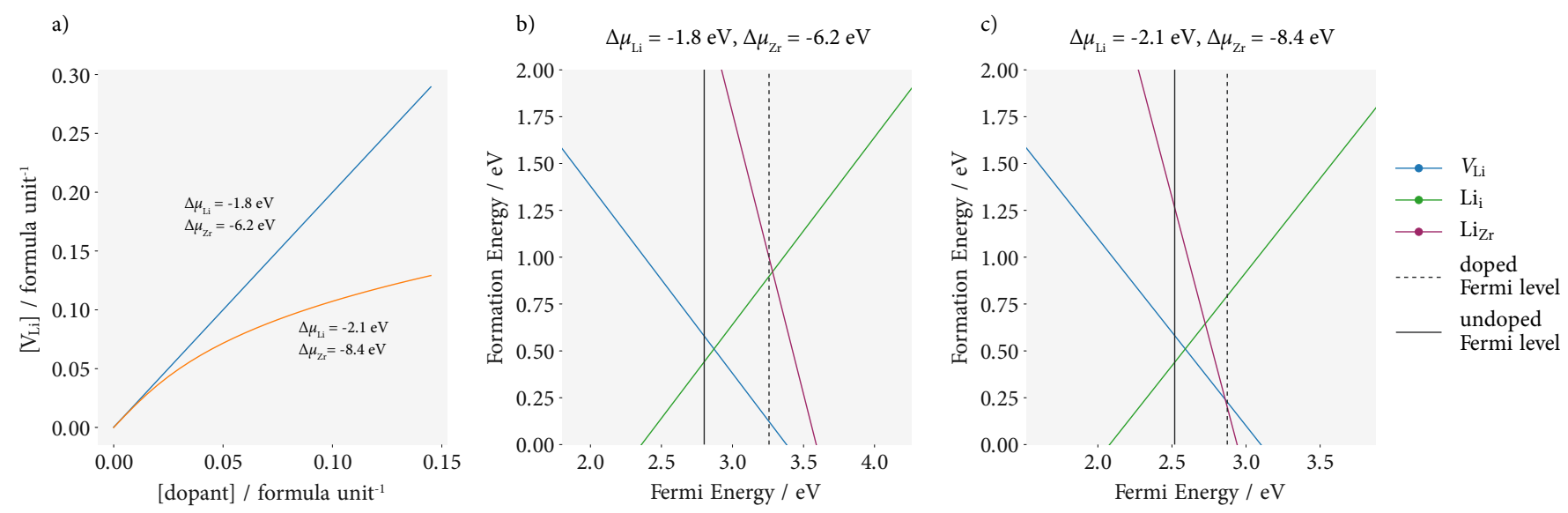

FIG. 5. (a) Increase in lithium vacancy concentration as the amount of dopant $M_{\mathrm{Li}}^{\bullet \bullet}$ per formula unit is increased for two different sets of chemical potentials. (b) and (c) Transition level diagrams for the two cases in (a). In each diagram we show the Fermi level position before, and after supervalent doping $\left(0.15 M_{\mathrm{Li}}^{\bullet \bullet}\right.$ per formula unit). For clarity, here we only show the $V_{\mathrm{Li}}, \mathrm{Li}_{\mathrm{i}}$, and $\mathrm{Li}_{\mathrm{Zr}}$ formation energies. Full transition level diagrams showing the formation energies of all native defects are provided in the SI, Fig. S7. Source: The data and code used to generate this figure, and the figure file, are available under the MIT licence as part of Ref. 71.

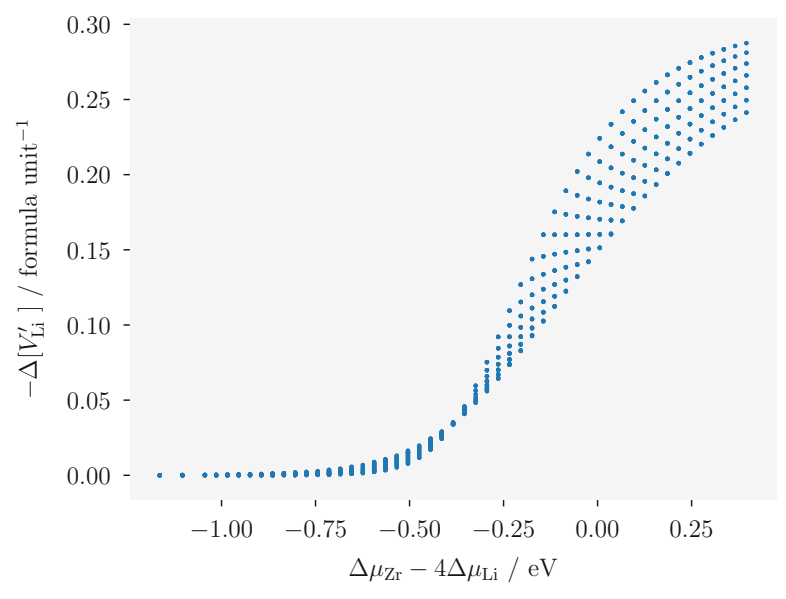

FIG. 6. Negative deviation of $\left[V^{\prime}{ }_{\mathrm{Li}}\right]$ concentration for doping at $\left[M_{\mathrm{Li}}^{\bullet \bullet}\right]=0.15$ per formula unit, relative to full charge compensation by $V_{\mathrm{Li}}$ (Eqn. 1), calculated at all points within the LLZO region of thermodynamic stability. Source: The data and code used to generate this figure, and the figure file, are available under the MIT licence as part of Ref. 71 .

though oxygen vacancies may readily diffuse during hightemperature sintering of samples.

We also find that the response to supervalent (donor) doping depends on thermodynamic conditions, and broadly depends on a balance between $\mathrm{Li}$ and $\mathrm{Zr}$ chemical potentials. Under relatively low $\Delta \mu_{\mathrm{Li}} /$ high $\Delta \mu_{\mathrm{Zr}}$ conditions, supervalent doping produces proportionate numbers of charge-compensating Li vacancies, as is often commonly assumed (e.g. Eqn. 4). Under relatively high $\Delta \mu_{\mathrm{Li}} /$ low $\Delta \mu_{\mathrm{Zr}}$ conditions, however, donor doping is chiefly compensated by $\mathrm{Li}_{\mathrm{Zr}}$ anitsites, and $\mathrm{Li}$ stoichiometries strongly deviate from those predicted by sim- ple "vacancy compensation" models. This result means that synthetic recipes that use doping to tune the Li stoichiometry in LLZO may not be able to assume direct compensation by Li vacancies, as the dominant compensating acceptor defect can vary with synthesis conditions. For this reason, we therefore recommend that experimental studies on the relationship between lithium stoichiometry and ionic conductivity directly measure lithium stoichiometries of as-synthesised materials, to obtain reliable values.

More broadly, this study illustrates how combining first-principles defect calculations with a grand-canonical thermodynamic model can provide a broad range of information on the stoichiometry, defect chemistry, and doping response of solid electrolytes. Accurate and quantitative calculations of defect chemistry have proven invaluable in understanding and optimising materials for a range of other applications, such as microelectronics, optoelectronics, and photovoltaics. ${ }^{86,92-95}$ We believe similarly detailed computational studies of the defect chemistry of battery materials may prove equally valuable, for example, in helping to develop rational synthetic schemes for optimising the properties of materials such as solid electrolytes.

A key challenge in developing accurate computational models of defect chemistry in solid lithium-ion electrolytes, however, is the question of how to treat the inherent Li-ion disorder that characterises many highly conducting electrolyte families. The defect formalism used here is formally valid only in systems with an ordered ground state. Eqn. 3, which relates defect concentrations to their formation energies, is derived by assuming the entropic contribution to defect chemical potentials is that of an ideal lattice-gas; i.e. the zero entropy reference state is a perfect ordered lattice. In an 
inherently disordered system, the entropy change when adding or removing a lithium ion will deviate from this ideal value, changing the relationship between defect concentration and formation energy. In addition, in a system with inherent lithium disorder, the energy change (formally enthalphy change) upon adding or removing a lithium ion is no longer given by the energy difference between single host and defect configurations (as in Eqn. 8). Instead, the energies of the host and defective systems should be computed as ensemble averages over all thermally accessible microstates. ${ }^{69}$ The development of quantitatively accurate models of defect equilibria in inherently disordered solid lithium-ion electrolytes is therefore expected to require advances in both the thermodynamic formalism used in constructing models and in the computational approaches used for calculating defect energies. Meeting these goals, however, brings the promise of a more precise understanding of defect chemistry of a broad range of solid electrolytes, and the potential for more accurate and practical tuning of their material properties through doping and controlled synthetic conditions.

\section{ACKNOWLEDGEMENTS}

A. G. S. acknowledges EPSRC for PhD funding, and thanks R. H. Brugge for stimulating discussions. D. O. S. acknowledges support from the EPSRC (EP/N001982/1 and EP/P00315X/1) and membership in the Materials Design Network. B. J. M. acknowledges support from the Royal Society (Grant No. UF130329). This work was supported by funding from the Faraday Institution (faraday.ac.uk; EP/S003053/1), grant no. FIRG003. Calculations were performed using the Balena High Performance Computing Service at the University of Bath, and the ARCHER supercomputer, through membership of the UK's HPC Materials Chemistry Consortium, funded by EPSRC grants EP/L000202 and EP/R029431.
* b.j.morgan@bath.ac.uk

1 M. M. Thackeray, C. Wolverton, and E. D. Isaacs, "Electrical energy storage for transportation - approaching the limits of, and going beyond, lithium-ion batteries," Energy Environ. Sci., vol. 5, no. 7, p. 7854, 2012.

2 J. C. Bachman, S. Muy, A. Grimaud, H. H. Chang, N. Pour, S. F. Lux, O. Paschos, F. Maglia, S. Lupart, P. Lamp, L. Giordano, and Y. Shao-Horn, "Inorganic Solid-State Electrolytes for Lithium Batteries: Mechanisms and Properties Governing Ion Conduction," Chem. Rev., vol. 116, no. 1, pp. 140-162, 2016.

3 V. Thangadurai, S. Narayanan, and D. Pinzaru, "Garnettype solid-state fast $\mathrm{Li}$ ion conductors for Li batteries: critical review," Chem. Soc. Rev., vol. 43, no. 13, p. 4714, 2014.

4 Z. Zhang, Y. Shao, B. Lotsch, Y.-S. Hu, H. Li, J. Janek, L. F. Nazar, C.-W. Nan, J. Maier, M. Armand, and L. Chen, "New horizons for inorganic solid state ion conductors," Energy Environ. Sci., vol. 11, no. 8, pp. 19451976, 2018.

${ }^{5}$ R. Chen, W. Qu, X. Guo, L. Li, and F. Wu, "The pursuit of solid-state electrolytes for lithium batteries: from comprehensive insight to emerging horizons," Mater. Horiz., vol. 3, pp. 487-516, 2016.

6 A. Manthiram, X. Yu, and S. Wang, "Lithium battery chemistries enabled by solid-state electrolytes," Nat. Rev. Mater., vol. 2, pp. 16103 EP -, 2017.

7 T. Famprikis, P. Canepa, J. A. Dawson, M. S. Islam, and C. Masquelier, "Fundamentals of inorganic solid-state electrolytes for batteries," Nat. Mater., vol. 21, pp. 1-14, 2019.

8 F. Zheng, M. Kotobuki, S. Song, M. O. Lai, and L. Lu, "Review on solid electrolytes for all-solid-state lithium-ion batteries," J. Power Sources, vol. 389, pp. 198 - 213, 2018.

9 Q. Liu, Z. Geng, C. Han, Y. Fu, S. Li, Y. bing He, F. Kang, and $\mathrm{B}$. Li, "Challenges and perspectives of garnet solid electrolytes for all solid-state lithium batteries," J. Power Sources, vol. 389, pp. 120 - 134, 2018.

10 Y. Li, J.-T. Han, C.-A. Wang, H. Xie, and J. B. Goodenough, "Optimizing $\mathrm{Li}^{+}$conductivity in a garnet framework," J. Mater. Chem., vol. 22, no. 30, p. 15357, 2012.

11 T. Thompson, S. Yu, L. Williams, R. D. Schmidt, R. Garcia-Mendez, J. Wolfenstine, J. L. Allen, E. Kioupakis, D. J. Siegel, and J. Sakamoto, "Electrochemical window of the Li-ion solid electrolyte $\mathrm{Li}_{7} \mathrm{La}_{3} \mathrm{Zr}_{2} \mathrm{O}_{12}$," ACS Energy Lett., vol. 2, no. 2, pp. 462 468, 2017.

12 N. J. Taylor, S. Stangeland-Molo, C. G. Haslam, A. Sharafi, T. Thompson, M. Wang, R. Garcia-Mendez, and J. Sakamoto, "Demonstration of high current densities and extended cycling in the garnet $\mathrm{Li}_{7} \mathrm{La}_{3} \mathrm{Zr}_{2} \mathrm{O}_{12}$ solid electrolyte," J. Power Sources, vol. 396, no. 6, pp. 314-318, 2018.

13 K. K. Fu, Y. Gong, B. Liu, Y. Zhu, S. Xu, Y. Yao, W. Luo, C. Wang, S. D. Lacey, J. Dai, Y. Chen, Y. Mo, E. Wachsman, and $\mathrm{L}$. Hu, "Toward garnet electrolytebased Li metal batteries: An ultrathin, highly effective, artificial solid-state electrolyte/metallic Li interface," Sci. Adv., vol. 3, no. 4, pp. 1-12, 2017.

${ }^{14} \mathrm{X}$. Yan, Z. Li, Z. Wen, and W. Han, "Li/ $\mathrm{Li}_{7} \mathrm{La}_{3} \mathrm{Zr}_{2} \mathrm{O}_{12} / \mathrm{LiFePO}_{4}$ all-solid-state battery with ultrathin nanoscale solid electrolyte," J. Phys. Chem. C, vol. 121, no. 3, pp. 1431-1435, 2017.

15 N. Zhao, W. Khokhar, Z. Bi, C. Shi, X. Guo, L.-Z. Fan, and C.-W. Nan, "Solid garnet batteries," Joule, pp. 1-10, Apr. 2019.

${ }^{16}$ H. Xie, J. A. Alonso, Y. Li, M. T. Fernández-Díaz, and J. B. Goodenough, "Lithium Distribution in AluminumFree Cubic $\mathrm{Li}_{7} \mathrm{La}_{3} \mathrm{Zr}_{2} \mathrm{O}_{12}$," Chem. Mater., vol. 23, pp. 3587-3589, Aug. 2011. 
17 W. G. Zeier, "Structural limitations for optimizing garnettype solid electrolytes: A perspective," Dalton Trans., vol. 43, no. 43, pp. 16133-16138, 2014.

18 S. Mukhopadhyay, T. Thompson, J. Sakamoto, A. Huq, J. Wolfenstine, J. L. Allen, N. Bernstein, D. A. Stewart, and M. D. Johannes, "Structure and Stoichiometry in Supervalent Doped $\mathrm{Li}_{7} \mathrm{La}_{3} \mathrm{Zr}_{2} \mathrm{O}_{12}$," Chem. Mater., vol. 27, no. 10 , pp. $3658-3665,2015$.

19 B. Kozinsky, S. A. Akhade, P. Hirel, A. Hashibon, C. Elsässer, P. Mehta, A. Logéat, and U. Eisele, "Effects of sublattice symmetry and frustration on ionic transport in garnet solid electrolytes," Phys. Rev. Lett., vol. 116, pp. 055901-5, Feb. 2016.

20 B. J. Morgan, "Lattice-geometry effects in garnet solid electrolytes: a lattice-gas Monte Carlo simulation study," R. Soc. Open Sci., vol. 4, pp. 170824-21, Nov. 2017.

21 M. Mottet, A. Marcolongo, T. Laino, and I. Tavernelli, "Doping in garnet-type electrolytes: Kinetic and thermodynamic effects from molecular dynamics simulations," Phys. Rev. Materials, vol. 3, p. 035403, Mar. 2019.

22 T. Thompson, J. Wolfenstine, J. L. Allen, M. Johannes, A. Huq, I. N. David, and J. Sakamoto, "Tetragonal vs. cubic phase stability in al - free ta doped $\mathrm{Li}_{7} \mathrm{La}_{3} \mathrm{Zr}_{2} \mathrm{O}_{12}$ (llzo)," J. Mater. Chem. A, vol. 2, pp. 13431-13436, 2014.

23 R. Murugan, V. Thangadurai, and W. Weppner, "Fast lithium ion conduction in garnet-type $\mathrm{Li}_{7} \mathrm{La}_{3} \mathrm{Zr}_{2} \mathrm{O}_{12}$," Angew. Chem. Int. Ed., vol. 46, pp. 7778-7781, 2007.

${ }^{24}$ C. A. Geiger, E. Alekseev, B. Lazic, M. Fisch, T. Armbruster, R. Langner, M. Fechtelkord, N. Kim, T. Pettke, and W. Weppner, "Crystal chemistry and stability of " $\mathrm{Li}_{7} \mathrm{La}_{3} \mathrm{Zr}_{2} \mathrm{O}_{12}$ " garnet: A fast lithium-ion conductor," Inorg Chem., vol. 50, pp. 1089-1097, Feb. 2011.

25 A. Kuhn, S. Narayanan, L. Spencer, G. Goward, V. Thangadurai, and M. Wilkening, "Li self-diffusion in garnet-type $\mathrm{Li}_{7} \mathrm{La}_{3} \mathrm{Zr}_{2} \mathrm{O}_{12}$ as probed directly by diffusioninduced ${ }^{7} \mathrm{Li}$ spin-lattice relaxation NMR spectroscopy," Phys. Rev. B, vol. 83, p. 094302, Mar. 2011.

26 M. Burbano, D. Carlier, F. Boucher, B. J. Morgan, and M. Salanne, "Sparse cyclic excitations explain the low ionic conductivity of stoichiometric $\mathrm{Li}_{7} \mathrm{La}_{3} \mathrm{Zr}_{2} \mathrm{O}_{12}$," Phys. Rev. Lett., vol. 116, pp. 135901-6, Mar. 2016.

27 D. Rettenwander, P. Blaha, R. Laskowski, K. Schwarz, P. Bottke, M. Wilkening, C. A. Geiger, and G. Amthauer, "DFT study of the role of $\mathrm{Al}^{3+}$ in the fast ion-conductor $\mathrm{Li}_{7-3 x} \mathrm{Al}_{x}^{3+} \mathrm{La}_{3} \mathrm{Zr}_{2} \mathrm{O}_{12}$ garnet," Chem. Mater., vol. 26, pp. 2617-2623, 2014.

28 N. Bernstein, M. D. Johannes, and K. Hoang, "Origin of the Structural Phase Transition in $\mathrm{Li}_{7} \mathrm{La}_{3} \mathrm{Zr}_{2} \mathrm{O}_{12}$," Phys. Rev. Lett., vol. 109, no. 20, 2012.

29 D. Rettenwander, R. Wagner, J. Langer, M. E. Maier, M. Wilkening, and G. Amthauer, "Crystal chemistry of " $\mathrm{Li}_{7} \mathrm{La}_{3} \mathrm{Zr}_{2} \mathrm{O}_{12}$ " garnet doped with $\mathrm{Al}, \mathrm{Ga}$, and Fe: a short review on local structures as revealed by NMR and Mößbauer spectroscopy studies," Eur. J. Mineral., vol. 28, pp. 619-629, 2016.

30 D. Rettenwander, C. A. Geiger, M. Tribus, P. Tropper, and G. Amthauer, "A synthesis and crystal chemical study of the fast ion conductor $\mathrm{Li}_{7-3 x} \mathrm{Ga}_{x} \mathrm{La}_{3} \mathrm{Zr}_{2} \mathrm{O}_{12}$ with $x=0.08$ to 0.84," Inorg. Chem., vol. 53, pp. 6264-6269, June 2014.

31 F. M. Pesci, R. H. Brugge, A. K. O. Hekselman, A. Cavallaro, R. J. Chater, and A. Aguadero, "Elucidating the role of dopants in the critical current density for dendrite formation in garnet electrolytes," J. Mater. Chem. A, vol. 6, pp. 19817-19827, 2018.
${ }^{32}$ R. H. Brugge, J. A. Kilner, and A. Aguadero, "Germanium as a donor dopant in garnet electrolytes," Solid State Ionics, vol. 337, pp. 154-160, May 2019.

33 S. R. Yeandel, B. J. Chapman, P. R. Slater, and P. Goddard, "Structure and Lithium-Ion Dynamics in FluorideDoped Cubic $\mathrm{Li}_{7} \mathrm{La}_{3} \mathrm{Zr}_{2} \mathrm{O}_{12}$ (LLZO) Garnet for Li SolidState Battery Applications," J. Phys. Chem. C, vol. 122, no. 49, pp. 27811-27819, 2018.

34 F. Kröger and H. Vink, "Relations between the concentrations of imperfections in crystalline solids," vol. 3 of Solid State Phys., pp. 307 - 435, Academic Press, 1956.

35 J. Wolfenstine, J. L. Allen, J. Read, and J. Sakamoto, "Chemical Stability of Cubic $\mathrm{Li}_{7} \mathrm{La}_{3} \mathrm{Zr}_{2} \mathrm{O}_{12}$ with molten lithium at elevated temperature," J. Mater. Sci., vol. 48, no. 17 , pp. 5846-5851, 2013.

36 M. Kubicek, A. Wachter-Welzl, D. Rettenwander, R. Wagner, S. Berendts, R. Uecker, G. Amthauer, H. Hutter, and J. Fleig, "Oxygen Vacancies in Fast Lithium-Ion Conducting Garnets," Chem. Mater., vol. 29, no. 17, pp. 7189-7196, 2017.

37 X. Zhan, S. Lai, M. P. Gobet, S. G. Greenbaum, and M. Shirpour, "Defect chemistry and electrical properties of garnet-type $\mathrm{Li}_{7} \mathrm{La}_{3} \mathrm{Zr}_{2} \mathrm{O}_{12}$," Phys. Chem. Chem. Phys., vol. 20, no. 3, pp. 1447-1459, 2018.

38 S. KC, R. C. Longo, K. Xiong, and K. Cho, "Point defects in garnet-type solid electrolyte $\left(\mathrm{c}-\mathrm{Li}_{7} \mathrm{La}_{3} \mathrm{Zr}_{2} \mathrm{O}_{12}\right)$ for Li-ion batteries," Solid State Ionics, vol. 261, pp. 100-105, 2014.

39 A. Moradabadi and P. Kaghazchi, "Defect chemistry in cubic $\mathrm{Li}_{6.25} \mathrm{Al}_{0.25} \mathrm{La}_{3} \mathrm{Zr}_{2} \mathrm{O}_{12}$ solid electrolyte: A density functional theory study," Solid State Ionics, vol. 338, pp. $74-$ 79, 2019.

40 S. B. Zhang and J. E. Northrup, "Chemical potential dependence of defect formation energies in GaAs: Application to Ga self-diffusion," Phys. Rev. Lett., vol. 67, pp. 2339-2342, 1991.

41 C. G. Van de Walle, D. B. Laks, G. F. Neumark, and S. T. Pantelides, "First-principles calculations of solubilities and doping limits: Li, Na, and $\mathrm{N}$ in ZnSe," Phys. Rev. B, vol. 47, pp. 9425-9434, Apr 1993.

42 C. Persson, Y.-J. Zhao, S. Lany, and A. Zunger, " $n$-type doping of $\mathrm{CuInSe}_{2}$ and $\mathrm{CuGaSe}_{2}, "$ Phys. Rev. B, vol. 72, p. 035211, Jul 2005.

43 C. Freysoldt, B. Grabowski, T. Hickel, J. Neugebauer, G. Kresse, A. Janotti, and C. G. Van de Walle, "Firstprinciples calculations for point defects in solids," Rev. Mod. Phys., vol. 86, pp. 253-305, Mar 2014.

44 C. Kittel and H. Kroemer, Thermal Physics (2nd Edition). W. H. Freeman, 1980.

45 J. Buckeridge, D. Jevdokimovs, C. R. Catlow, and A. A. Sokol, "Nonstoichiometry and Weyl fermionic behavior in TaAs," Phys. Rev. B, vol. 94, no. 18, 2016.

46 J. Buckeridge, "Equilibrium point defect and charge carrier concentrations in a material determined through calculation of the self-consistent Fermi energy," Comput. Phys. Commun., 2019. In Press, https://doi.org/10.1016/j.cpc.2019.06.017.

47 F. A. G. Daza, M. R. Bonilla, A. Llordés, J. Carrasco, and E. Akhmatskaya, "Atomistic insight into ion transport and conductivity in $\mathrm{Ga} / \mathrm{Al}$-substituted $\mathrm{Li}_{7} \mathrm{La}_{3} \mathrm{Zr}_{2} \mathrm{O}_{12}$ solid electrolytes," ACS Appl. Mater. Int., vol. 11, no. 1, pp. 753-765, 2018.

48 S. Lany and A. Zunger, "Assessment of correction methods for the band-gap problem and for finite-size effects in supercell defect calculations: Case studies for $\mathrm{ZnO}$ and 
GaAs," Phys. Rev. B, vol. 78, no. 23, pp. 17-20, 2008.

49 S. T. Murphy and N. D. M. Hine, "Anisotropic charge screening and supercell size convergence of defect formation energies," Phys. Rev. B, vol. 87, p. 094111, 2013.

50 J. Perdew, A. Ruzsinszky, G. Csonka, O. Vydrov, G. Scuseria, L. Constantin, X. Zhou, and K. Burke, "Restoring the Density Gradient-Expansion for Exchange in Solids and Surfaces," Phys. Rev. Lett., vol. 100, no. 13, p. 136406 , 2008.

51 P. Canepa, J. A. Dawson, G. Sai Gautam, J. M. Statham, S. C. Parker, and M. S. Islam, "Particle morphology and lithium segregation to surfaces of the $\mathrm{Li}_{7} \mathrm{La}_{3} \mathrm{Zr}_{2} \mathrm{O}_{12}$ solid Electrolyte," Chem. Mater., vol. 30, no. 9, pp. 3019-3027, 2018.

52 J. Buckeridge, D. O. Scanlon, A. Walsh, and C. R. Catlow, "Automated procedure to determine the thermodynamic stability of a material and the range of chemical potentials necessary for its formation relative to competing phases and compounds," Comput. Phys. Comm., vol. 185, no. 1, pp. 330-338, 2014.

${ }^{53}$ G. Kresse and J. Hafner, "Ab initio molecular dynamics for liquid metals," Phys. Rev. B, vol. 47, no. 1, pp. 558-561, 1993.

${ }^{54}$ G. Kresse and J. Hafner, "Ab initio molecular-dynamics simulation of the liquid-metalamorphous- semiconductor transition in germanium," Phys. Rev. B, vol. 49, no. 20, pp. 14251-14269, 1994.

${ }^{55}$ G. Kresse and J. Furthmüller, "Efficiency of ab-initio total energy calculations for metals and semiconductors using a plane-wave basis set," Comput. Mater. Sci., vol. 6, no. 1, pp. 15-50, 1996.

${ }^{56}$ G. Kresse and J. Furthmüller, "Efficient iterative schemes for ab initio total-energy calculations using a plane-wave basis set," Phys. Rev. B., vol. 54, no. 16, pp. 11169-11186, 1996.

57 P. E. Blöchl, "Projector augmented-wave method," Phys. Rev. B, vol. 50, no. 24, pp. 17953-17979, 1994.

${ }^{58}$ F. D. Murnaghan, "The Compressibility of Media under Extreme Pressures," Proc. Natl. Acad. Sci., vol. 30, no. 9, pp. 244-247, 1944.

59 A. V. Krukau, O. A. Vydrov, A. F. Izmaylov, and G. E. Scuseria, "Influence of the exchange screening parameter on the performance of screened hybrid functionals," $J$. Chem. Phys., vol. 125, no. 22, 2006.

60 J. Awaka, N. Kijima, H. Hayakawa, and J. Akimoto, "Synthesis and structure analysis of tetragonal $\mathrm{Li}_{7} \mathrm{La}_{3} \mathrm{Zr}_{2} \mathrm{O}_{12}$ with the garnet-related type structure," J. Solid State Chem., vol. 182, pp. 2046-2052, July 2009

61 A. Logéat, T. Köhler, U. Eisele, B. Stiaszny, A. Harzer, M. Tovar, A. Senyshyn, H. Ehrenberg, and B. Kozinsky, "From order to disorder: The structure of lithiumconducting garnets $\mathrm{Li}_{7-x} \mathrm{La}_{3} \mathrm{Ta}_{x} \mathrm{Zr}_{2-x} \mathrm{O}_{12}$ ( $\left.x=0-2\right)$," Solid State Ionics, vol. 206, pp. 33 - 38, 2012.

${ }^{62}$ F. Oba, M. Choi, A. Togo, and I. Tanaka, "Point defects in ZnO: an approach from first principles," Sci. Technol. Adv. Mater., vol. 12, pp. 034302-034302, May 2011.

63 D. O. Scanlon, B. J. Morgan, G. W. Watson, and A. Walsh, "Acceptor levels in $p$-type $\mathrm{Cu}_{2} \mathrm{O}$ : Rationalizing theory and experiment," Phys. Rev. Lett., vol. 103, no. 9, p. 096405, 2009.

64 A. Janotti and C. G. Van de Walle, "LDA $+U$ and hybrid functional calculations for defects in $\mathrm{ZnO}, \mathrm{SnO}_{2}$, and $\mathrm{TiO}_{2}$," Phys. Stat. Sol. B, vol. 248, no. 4, pp. 799-804, 2011.
65 P. Deák, B. Aradi, and T. Frauenheim, "Polaronic effects in $\mathrm{TiO}_{2}$ calculated by the HSE06 hybrid functional: Dopant passivation by carrier self-trapping," Phys. Rev. B, vol. 83, p. 155207, Apr. 2011.

66 B. J. Morgan and G. W. Watson, "A DFT $+U$ description of oxygen vacancies at the $\mathrm{TiO}_{2}$ rutile (110) surface," Surf. Sci., vol. 601, pp. 5034-5041, 2007.

67 D. O. Scanlon, B. J. Morgan, and G. W. Watson, "Modeling the polaronic nature of $p$-type defects in $\mathrm{Cu}_{2} \mathrm{O}$ : The failure of GGA and GGA+U," J. Chem. Phys., vol. 131, p. 124703, 2009.

68 B. J. Morgan, D. O. Scanlon, and G. W. Watson, "The use of the " $+U$ " correction in describing defect states at metal oxide surfaces: Oxygen vacancies on $\mathrm{CeO}_{2}$ and $\mathrm{TiO}_{2}$, and Li-doping of MgO," e-J. Surf. Sci. Nanotech., vol. 7, pp. 389-394, 2009.

69 P. Gorai, H. Long, E. Jones, S. Santhanagopalan, and V. Stefanović, "Defect chemistry of disordered solid-state electrolyte $\mathrm{Li}_{10} \mathrm{GeP}_{2} \mathrm{~S}_{12}$," chemRxiv, 2019.

70 https://researchdata.bath.ac.uk/id/eprint/691.

71 https://github.com/alexsquires/native-defects-in-llzo.

72 J. D. Hunter, "Matplotlib: A 2d graphics environment," Comput. Sci. Eng., vol. 9, no. 3, pp. 90-95, 2007.

73 S. v. d. Walt, S. C. Colbert, and G. Varoquaux, "The numpy array: A structure for efficient numerical computation," Comput. Sci. Eng., vol. 13, pp. 22-30, Mar. 2011.

74 W. McKinney, "Data structures for statistical computing in Python," in Proceedings of the 9th Python in Science Conference (S. van der Walt and J. Millman, eds.), pp. 51 $-56,2010$.

75 S. P. Ong, W. D. Richards, A. Jain, G. Hautier, M. Kocher, S. Cholia, D. Gunter, V. L. Chevrier, K. A. Persson, and G. Ceder, "Python materials genomics (pymatgen): A robust, open-source python library for materials analysis," Comp. Mater. Sci., vol. 68, pp. 314-319, 2013.

76 T. E. Oliphant, "Python for scientific computing," Comput. Sci. Eng, vol. 9, no. 3, pp. 10-20, 2007.

77 C. O. da Costa-Luis, "tqdm: A fast, extensible progress meter for Python and CLI," JOSS, vol. 4, p. 1277, 52019.

78 B. J. Morgan, "vasppy: A Python suite for manipulating VASP input and output," 2018. https://github.com/bjmorgan/vasppy.

B. J. Morgan, "vasppy," May 2019.

79 J. L. Allen, J. Wolfenstine, E. Rangasamy, and J. Sakamoto, "Effect of substitution (Ta, Al, Ga) on the conductivity of $\mathrm{Li}_{7} \mathrm{La}_{3} \mathrm{Zr}_{2} \mathrm{O}_{12}$," J. Power Sources, vol. 206, pp. 315-319, May 2012.

80 J. Neugebauer and C. G. Van de Walle, "Theory of point defects and complexes in gan," MRS Proceedings, vol. 395, p. $645,1995$.

81 Calculations performed using PBEsol in a 192 atom conventional cell indicate that $V_{\mathrm{Li}}-\mathrm{Li}_{\mathrm{i}}$ pairs separated by more than $4.2 \mathrm{eV}$ give a converged "well-separated" energy.

82 The calculated formation energy of a $V_{\mathrm{Li}}-\mathrm{Li}_{\mathrm{i}}$ pair at $4.16 \AA$ is $0.83 \mathrm{eV}$, and at $6.13 \AA$ is $0.92 \mathrm{eV}$.

83 B. J. Morgan and G. W. Watson, "Intrinsic $n$-type defect formation in $\mathrm{TiO}_{2}$ : A comparison of rutile and anatase from GGA $+U$ calculations," J. Phys. Chem. C, vol. 114, pp. 2321-2328, 2010.

${ }^{84}$ G. Henkelman, B. P. Uberuaga, and H. Jónsson, "Climbing image nudged elastic band method for finding saddle points and minimum energy paths," J. Chem. Phys., vol. 113, no. 22, pp. 9901-9904, 2000. 
85 M. Cherry, M. Islam, and C. Catlow, "Oxygen Ion Migration in Perovskite-Type Oxides," J. Sol. Stat. Chem., vol. 118, no. 1, pp. 125-132, 1995.

${ }^{86}$ F. H. Taylor, J. Buckeridge, and C. R. A. Catlow, "Defects and oxide ion migration in the solid oxide fuel cell cathode material $\mathrm{LaFeO}_{3}$," Chem. Mater., vol. 28, no. 22, pp. 82108220, 2016.

87 R. D. Shannon, "Revised effective ionic radii and systematic studies of interatomic distances in halides and chalcogenides," Acta Crystallogr., Sect. A:, vol. 32, pp. 751-767, Sep 1976.

88 R. Murugan, V. Thangadurai, and W. Weppner, "Lithium ion conductivity of $\mathrm{Li}_{5+x} \mathrm{Ba}_{x} \mathrm{La}_{3-x} \mathrm{Ta}_{2} \mathrm{O}_{12}(x=0-2)$ with garnet-related structure in dependence of the barium content," Ionics, vol. 13, pp. 195-203, June 2007.

89 E. J. Cussen, "Structure and ionic conductivity in lithium garnets," J. Mater. Chem., vol. 20, no. 25, pp. 5167-5173, 2010.

90 S. Ohta, T. Kobayashi, and T. Asaoka, "High lithium ionic conductivity in the garnet-type oxide $\mathrm{Li}_{7-x} \mathrm{La}_{3}\left(\mathrm{Zr}_{2-x}\right.$, $\left.\mathrm{Nb}_{x}\right) \mathrm{O}_{12}(x=0-2), "$ J. Power Sources, vol. 196, pp. 3342-
3345, Mar. 2011.

91 Y. Wang and W. Lai, "High ionic conductivity lithium garnet oxides of $\mathrm{Li}_{7-x} \mathrm{La}_{3} \mathrm{Zr}_{2-x} \mathrm{Ta}_{x} \mathrm{O}_{12}$ compositions," Electrochem. Sol. Stat. Lett., vol. 15, no. 5, p. A68, 2012.

92 A. Goyal, P. Gorai, E. S. Toberer, and V. Stevanović, "First-principles calculation of intrinsic defect chemistry and self-doping in PbTe," npj Comput. Mater., vol. 3, no. 1, pp. 1-9, 2017.

93 A. M. Ganose, S. Matsumoto, J. Buckeridge, and D. O. Scanlon, "Defect Engineering of Earth-Abundant Solar Absorbers BiSI and BiSeI," Chem. Mater., vol. 30, no. 11, pp. 3827-3835, 2018.

94 B. J. Morgan and G. W. Watson, "Polaronic trapping of electrons and holes in anatase $\mathrm{TiO}_{2}$," Phys. Rev. B, vol. 80, no. 233102, p. 233102, 2009.

95 D. O. Scanlon, A. B. Kehoe, G. W. Watson, M. O. Jones, W. I. F. David, D. J. Payne, R. G. Egdell, P. P. Edwards, and A. Walsh, "Nature of the band gap and origin of the conductivity of $\mathrm{PbO}_{2}$ revealed by theory and experiment," Phys. Rev. Lett., vol. 107, p. 246402, 2011. 